\title{
Comparison of Temporalis Myofascial Flap vs Dermal Graft as an Interpositional Material in Treatment of Temporomandibular Joint Ankylosis: A Case Series
}

\author{
Alankrita Sisodia ${ }^{1}$, Ruchika Tiwari ${ }^{2}$, Vikas K Singh ${ }^{3}$, Amit Bhamboo $^{4}$, Yashpal Gurjar ${ }^{5}$, Sonali Mishra ${ }^{6}$
}

\begin{abstract}
Temporomandibular ankylosis is a condition that affects the function and esthetics of a person. Various options are available as interpositional grafts to prevent recurrence of ankylosis, e.g., meniscus, dura skin, fascia, fat, muscle, cartilage, alloplastic materials, and xenografts. The merits and demerits of the dermal fat graft as an interpositional material following gap arthroplasty were compared with temporalis fascia interposition in this study. Twenty patients with TMJ ankylosis (three patients had bilateral TMJ ankylosis) were randomly divided into two groups of 10 patients each; in group I, interposition with the dermis-fat graft that was taken from the suprapubic region was done. Patients in the control group had an interposition with temporalis fascia and muscle from the same surgical site. All the patients were evaluated by age, sex, clinical features, etiology, and postsurgical complications. The age of all the patients was between 2 years and 11 years. Postoperatively and during follow-up, the interincisal mouth opening was adequate with proper healing of the donor site. Conclusion states that the use of the dermal fat graft shows minimum donor site morbidity and proves to be an effective interposition material to prevent recurrence of TMJ ankylosis.

Keywords: Ankylosis, Arthroplasty, Dermis-fat graft, Temporalis myofascial graft, Temporomandibular joint. Journal of Mahatma Gandhi University of Medical Sciences \& Technology (2019): 10.5005/jp-journals-10057-0116
\end{abstract}

\section{INTRODUCTION}

Temporomandibular ankylosis is a condition that is characterized by pathological changes occurring in the temporomandibular joint (TMJ) surface ${ }^{1}$ with limitation of movement of the mandible, which results in significantly decreased mouth opening; it also restricts mandibular movements such as protrusion and lateral excursion to varying degrees depending on the type and extent of the involvement. It therefore causes problems in speech, chewing, cosmetic abnormality, and poor oral hygiene. The management of such a disability aims at reinstating the normal function of mandible to prevent relapse of the condition, to get back the normal appearance of the patient, and to achieve normal occlusion and growth. ${ }^{1-4}$ Gap arthroplasty followed by interpositional grafting is the gold standard for primary surgical management of TMJ ankylosis. Various options are available as interpositional grafts to prevent recurrence of ankylosis, e.g., temporomandibular meniscus, dura skin, fascia lata, fat, muscle, cartilage, alloplastic materials, and xenografts. Also the dermal fat graft has been used for the treatment of palatal fistulas and augmentation rhinoplasties in the past. ${ }^{5}$ This study has assessed the merits and demerits of the dermal fat graft and juxtaposed it with the standard method of treatment by temporalis myofascial flap to overcome the problem of relapse.

\section{Materials and Methods}

Twenty patients with restricted mouth opening due to TMJ ankylosis who visited the Outpatient Department of Oral and Maxillofacial Surgery, Mahatma Gandhi Dental College and Hospital, Jaipur, between the period 2018 and 2020 were randomly selected. Informed consent and detailed history of the ailment were recorded. All the patients were examined for deviation of chin, facial asymmetry, midline shift, scars, occlusion, mouth opening, and any evidence of lateral, protrusive, or retrusive
${ }^{1-6}$ Department of Oral and Maxillofacial Surgery, Mahatma Gandhi Dental College and Hospital, Mahatma Gandhi University of Medical Sciences and Technology, Jaipur, Rajasthan, India

Corresponding Author: Alankrita Sisodia, Department of Oral and Maxillofacial Surgery, Mahatma Gandhi Dental College and Hospital, Mahatma Gandhi University of Medical Sciences and Technology, Jaipur, Rajasthan, India, Phone: +91 7737227231, e-mail: milisisodia3@ gmail.com

How to cite this article: Sisodia A, Tiwari R, Singh VK, et al. Comparison of Temporalis Myofascial Flap vs Dermal Graft as an Interpositional Material in Treatment of Temporomandibular Joint Ankylosis: A Case Series. J Mahatma Gandhi Univ Med Sci Tech 2019;4(3):77-80.

Source of support: Nil

Conflict of interest: None

movements. Orthopantomogram (OPG), CT scans and routine blood investigations were obtained for all patients. Ten patients were allocated to each group. All the patients were operated under general anesthesia wherein the joint was exposed through Thoma's angulated incision in group I and Al-Kayat Bramley incision in group II and a gap arthroplasty was performed. The ankylosed segment was separated following osteotomy cuts to create a minimum of 1.5 $\mathrm{cm}$ gap (Fig. 1). Minimum mouth opening of $35 \mathrm{~mm}$ was achieved intraoperatively and coronoidectomy was performed in the patients in which adequate mouth opening could not be achieved. A temporalis myofascial pedicled flap was dissected and passed under the zygomatic arch (Fig. 2) to place in the gap arthroplasty in the second group of patients. In the first group, the dermal fat graft was secured by making an elliptical incision of about $6 \times 3$ $\mathrm{cm}$ in the skin of the suprapubic region to gain a graft volume of approximately $5-20 \mathrm{~mL}$ (Figs 3 and 4). This volume was about $20 \%$ more than that actually required for the interposition. The donor site 


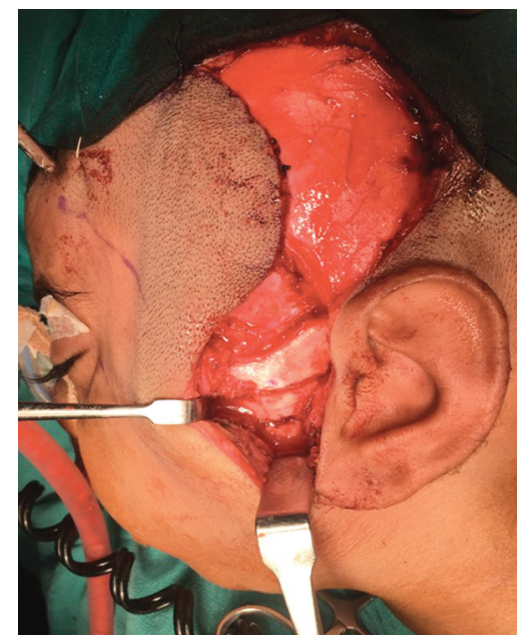

Fig. 1: Osteotomized segment of bone

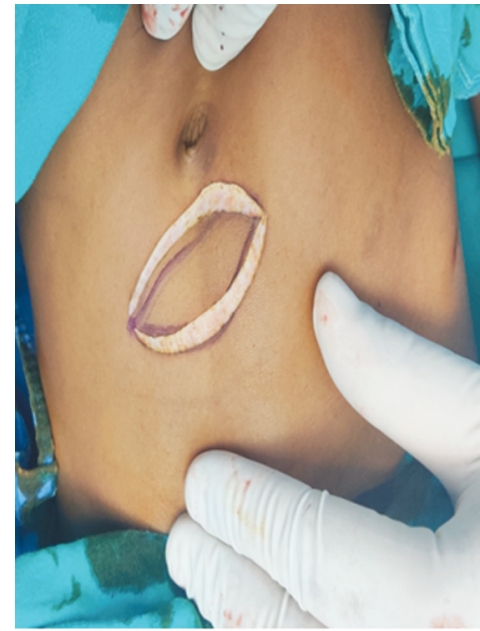

Fig. 3: Incision for harvest of dermis-fat graft

wound margins were undermined followed by a layer-wise closure. The subcutaneous fat was neatly trimmed to fill the gap making sure that all the dead spaces were eliminated. The graft was folded and sutured to the surrounding soft tissues so that the epithelial layer was apposed with the surface of glenoid fossa (Fig. 5). A suction drain was placed in the region and wound was repaired in layers. Physiotherapy was commenced 7 days postoperatively for mouth opening and continued thereafter to maintain the improved mouth opening. The patients were evaluated for maximum mouth opening, involvement of the facial nerve, and recurrence of ankylosis. Swelling, postoperative paresthesia, pain (VAS scale), and infection were assessed at both donor and recipient sites. The Student's $t$-test and the Fisher's exact test were used to study the differences between the two groups.

\section{Results}

Twenty patients all under the age of 20 years were studied with TMJ ankylosis. Among these, ten patients were treated with placement of dermal fat grafts, and in ten patients the temporalis fascia and muscle were used for interposition. Trauma was the most common cause of ankylosis in most of the patients. Most common clinical finding was retruded chin followed by midline shift and

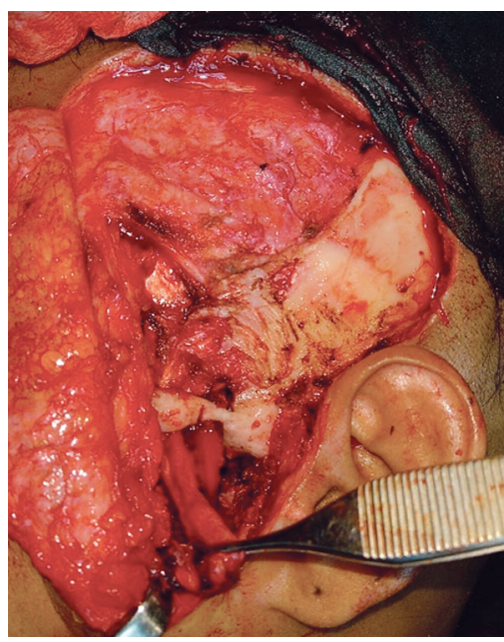

Fig. 2: Temporalis myofascial flap harvested

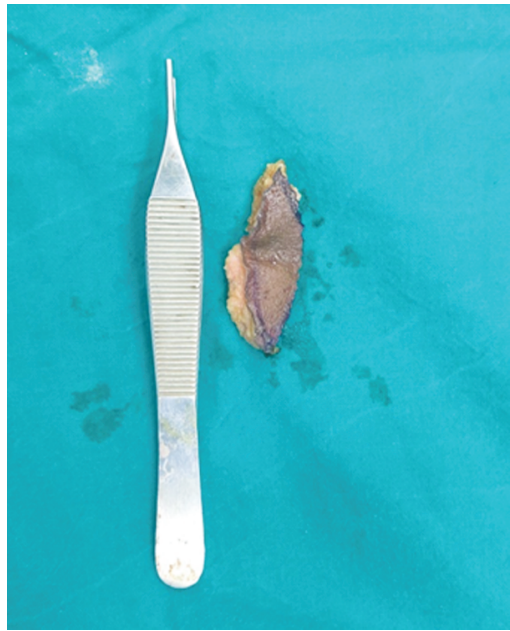

Fig. 4: Harvested dermis graft

facial asymmetry. Postoperatively, the interincisal mouth opening improved by a considerable amount (Figs 6 to 8). There was no significant difference in mouth opening in-between the two groups postoperatively, but there was higher mean in the dermal fat group (Table 1). However, postoperatively at all the intervals the mean value of mouth opening in the dermal fat group was remarkably more than in the other group. There was more scarring of the muscle in the temporalis group as the graft had been harvested from the same surgical site. In the two groups, there was no notable difference in the mean scores for pain and swelling. There was relapse after a period of 16 months in one patient in the temporalis fascia group and ankylosis recurred, the reason being that temporalis myofasical flap is thin and due to lack of physiotherapy the bony reunion may occur in the surgically created gap.

\section{Discussion}

There have been controversies related to the advantage of either gap or interposition arthroplasty (type of graft to be interposed). Topazian and Dimitroulis highlighted the advantage of interpositional grafting over gap arthroplasty in different studies as an effective means of reducing relapse of ankylosis $(53 \%$ recurrence rate in cases treated by gap arthroplasty). ${ }^{6}$ None of the 


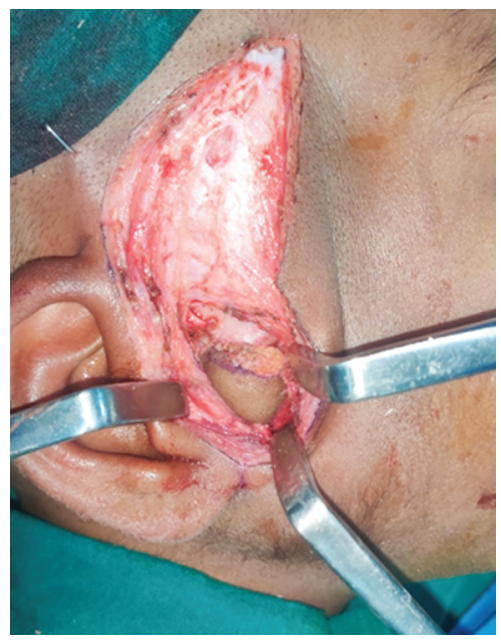

Fig. 5: Graft placed in the created gap

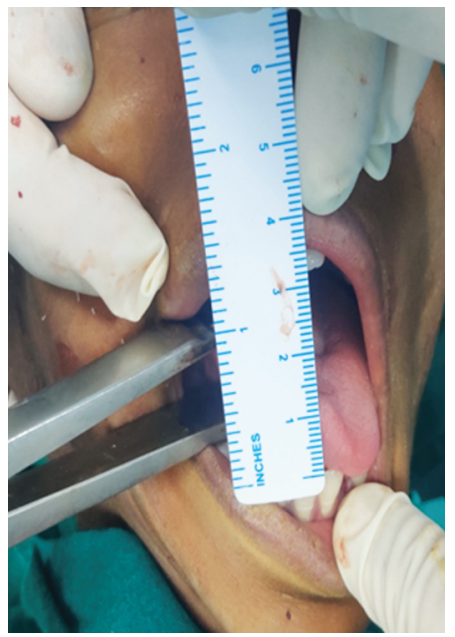

Fig. 7: Intraoperative mouth opening of $32 \mathrm{~mm}$

interpositional graft materials can be considered as the ideal choice as the cartilage tends to fibrose and calcify, the muscle shrinks and gets fibrosed, and the fascia is deficient in bulk, while the alloplastic implants disintegrate under functional loads. Temporalis myofascial flaps are the most chosen grafts but as the temporalis muscle is dissected, scar contracture at the donor site aggravates the trismus further. Reconstruction following gap arthroplasty is important for restoration of function to avoid a distressing facial deformity and open bite by loss of vertical dimension ${ }^{7}$ in cases of bilateral ankylosis. Along with removal of the ankylosed bone segment, appropriate physiotherapy and regular follow-up are important steps to avoid chances of relapse. ${ }^{8}$ A dermal graft has an advantage over fascia, muscle, cartilage, or fat-fascia transplants as it has superior viability, can be harvested in any desired quantity, and is easily adaptable to any surface. ${ }^{9}$ The fat-fascia transplants resorb at a rate of $20-100 \%$ whereas this dermal fat graft resorbs at a rate of about $15 \%$ only. Therefore, this autogenous dermal fat graft can withstand the stress of reconstructing a new, functional TMJ without any loss of posterior vertical dimension. ${ }^{10}$ Other indications for the use of dermal-fat graft are closure of palatal fistulas, correction of facial defects, ${ }_{11}^{11}$ for augmentation rhinoplasties, cases of orbital implant ${ }^{12}$ in enophthalmic sockets in children, to restore the concave deformity following parotidectomy. ${ }^{5}$

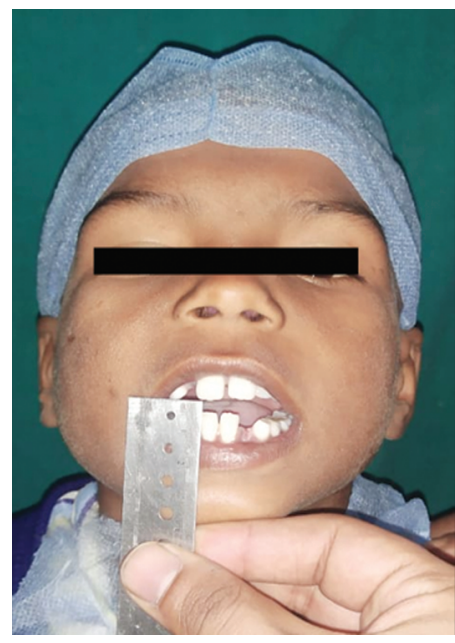

Fig. 6: Preoperative mouth opening $(4 \mathrm{~mm})$

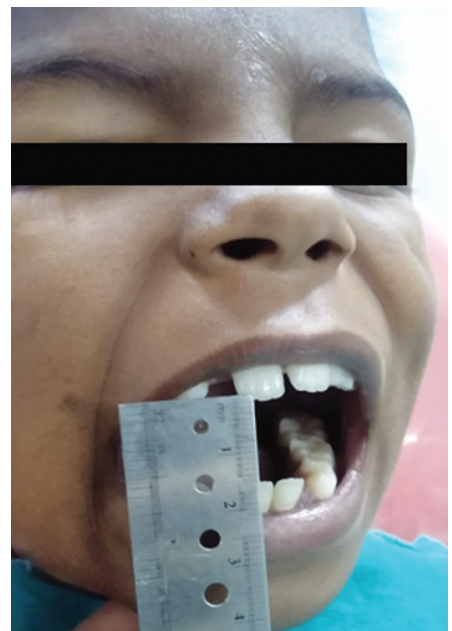

Fig. 8: Postoperative mouth opening of $22 \mathrm{~mm}$

Table 1: Result comparison between the two groups

\begin{tabular}{lccl}
\hline Time interval & Group I & Group II & p value \\
\hline Mean mouth opening (mm) & \multicolumn{2}{c}{ Data are mean (SD) } \\
Preoperative & $3.9(3.2)$ & $2.0(3.5)$ & 0.27 \\
Immediate postoperative & $33.8(4.3)$ & $27.6(5.8)$ & 0.03 \\
6 months' postoperative & $33.3(4.4)$ & $25.9(5.4)$ & 0.008 \\
Pain score (0-3) & & & \\
Immediate postoperative & $2.5(0.8)$ & $2.7(0.5)$ & 0.59 \\
1 week postoperative & $1.4(0.5)$ & $1.3(0.5)$ & 0.87 \\
6 months postoperative & 0 & 0 & - \\
Swelling score (0-3) & & & \\
Immediate postoperative & $2.0(0.5)$ & $2.1(0.5)$ & 0.69 \\
1 week postoperative & 0 & 0 & - \\
6 months postoperative & 0 & 0 & - \\
\hline
\end{tabular}

\section{ConCLUSION}

Interpositional arthroplasty using the dermis-fat graft in the management of TMJ ankylosis is a safe and effective procedure especially in children. The tendency of reankylosis is more common 
among children due to poor patient compliance. In comparison with the temporalis flap, the patients with the dermis fat graft were able to perform better jaw movements and physiotherapy. The advantages of dermis-fat graft are that it minimizes the incidence of excessive joint fibrosis and heterotopic calcification and thus provides improved range of motion. Harvesting of the graft is a very brief and easy procedure with minimal morbidity and the wound being in the anatomical skin crease is accepted cosmetically. The results obtained in this study are highly satisfactory and encouraging, supporting the role of the dermis fat graft as an interpositional material in TMJ ankylosis cases especially in children. However, a large sample size and a longer follow-up period are required to consolidate the findings.

\section{References}

1. Rowe NL. Ankylosis of the temporomandibular joint. J R Coll Surg Edinb 1982;27:67-79.

2. Rowe NL. Ankylosis of the temporomandibular joint. Part 2. J R Coll Surg Edinb 1982;27:167-173.

3. Rowe NL. Ankylosis of the temporomandibular joint. Part 3. J R Coll Surg Edinb 1982;27:209-218.

4. El Sheikh MM. Temporomandibular joint ankylosis: the Egyptian experience. Ann R Coll Surg Engl 1999;81:12-18.
5. Dimitroulis $G$. The interpositional dermis fat graft in the management of temporomandibular joint ankylosis. Int J Oral Maxillofac Surg 2004;33(8):755-760. DOI: 10.1016/j.ijom.2004.01.012.

6. Topazian RG. Comparison of gap and interposition arthroplasty in the treatment of temporomandibular joint ankylosis. J Oral Surg 1966;24:405-409.

7. Hinds EC, Pleasants JE. Reconstruction of the temporomandibular joint. Br J Plast Surg 1953;5:105-109.

8. Erol B, Tanrikulu R, Gorgun B. A clinical study on ankylosis of the temporomandibular joint. J Craniomaxillofac Surg 2006;34(2): 100-106. DOI: 10.1016/j.jcms.2005.07.008.

9. Georgiade N, Altany F, Pickrell K. An experimental and clinical evaluation of autogenous dermal grafts used in the treatment of TMJ ankylosis. Plast Reconstr Surg 1957;19(4):321-336. DOI: 10.1097/00006534-195704000-00004.

10. Piero C, Alessandro A, Giorgio S, et al. Combined surgical therapy of temporomandibular ankylosis and secondary deformity using intraoral distraction. J Craniofac Surg 2002;13(3):401-409. DOI: 10.1097/00001665-200205000-00008.

11. Schuessler W, Steffanoff D. Dermal grafts for correction of facial defects. Plast Reconstr Surg 1949;4:341-343.

12. Guberina C, Hornblass A, Meltzer MA, et al. Autogenous dermis fat orbital implantation. Arch Ophthalmol 1983;101(10):1586-1590. DOI: 10.1001/archopht.1983.01040020588018. 